\title{
Cardiotoxicity evaluation using magnetic resonance imaging in breast Cancer patients (CareBest): study protocol for a prospective trial
}

Yoo Jin Hong ${ }^{1}$, Gun Min Kim², Kyunghwa Han, Pan Ki Kim, Su An Lee, Eunkyung An¹, Ji Yeon Lee, Hye-Jeong Lee', Jin Hur ${ }^{1}$, Young Jin Kim¹, Min Jung Kim ${ }^{1{ }^{*+}}$ and Byoung Wook Choi ${ }^{1{ }^{*+}}$ (D)

\begin{abstract}
Background: Cardiovascular disease is second only to cancer recurrence as a determinant of lifespan in cancer survivors, and cancer therapy-related cardiac dysfunction is a clinically important risk factor. We aim to investigate the use of cardiac magnetic resonance imaging (MRI) to evaluate early tissue changes and perform functional assessment of chemo- and radiation-induced cardiotoxicity and to identify MRI prognostic indicators of cardiotoxicity in breast cancer patients.

Methods: A 3-min cardiac imaging protocol will be added to the breast MRI examination to diagnose cardiotoxicity in breast cancer patients. Standardized MRI-based evaluation of breast cancer and the left ventricular myocardium will be performed at baseline and at 3, 6, and 12 months and 2 years or more after cancer treatment. We will analyze both ventricular volume and ejection fraction (EF), strain of left ventricle (LV), native T1, extracellular volume fraction (ECV), and T2 values acquired in the mid LV.

Discussion: The primary result of this study will be the comparison of the prognostic value of MRI parameters (native T1, ECV, both ventricular systolic function and LV strain) for cardiotoxicity. The endpoint is defined as the occurrence of a major adverse cardiac event (MACE). The secondary outcome will be an assessment of the temporal relationships between contractile dysfunction and microstructural injury over 4 years using MRI. This study will assess the usefulness of quantitative MRI to diagnose cardiotoxicity and will clarify the temporal relationships between contractile dysfunction and microstructural injury of the LV myocardium using MRI during breast cancer treatment.
\end{abstract}

Trial registration: The protocol was registered at clinicaltrials.gov (Clinical trial no. NCT03301389) on October 4, 2017. Keywords: Magnetic resonance imaging, Cardiotoxicity, Native T1, Extracellular volume fraction, T1 mapping

\footnotetext{
* Correspondence: MINES@yuhs.ac; bchoi305@gmail.com; bchoi@yuhs.ac ${ }^{\dagger}$ Min Jung Kim and Byoung Wook Choi contributed equally to this work. 'Department of Radiology and Research Institute of Radiological Science, Severance Hospital, Yonsei University College of Medicine, 50 Yonsei-ro, Seodaemun-gu, Seoul 120-752, South Korea

Full list of author information is available at the end of the article
}

C C The Author(s). 2020 Open Access This article is licensed under a Creative Commons Attribution 4.0 International License, which permits use, sharing, adaptation, distribution and reproduction in any medium or format, as long as you give appropriate credit to the original author(s) and the source, provide a link to the Creative Commons licence, and indicate if changes were made. The images or other third party material in this article are included in the article's Creative Commons licence, unless indicated otherwise in a credit line to the material. If material is not included in the article's Creative Commons licence and your intended use is not permitted by statutory regulation or exceeds the permitted use, you will need to obtain permission directly from the copyright holder. To view a copy of this licence, visit http://creativecommons.org/licenses/by/4.0/. The Creative Commons Public Domain Dedication waiver (http://creativecommons.org/publicdomain/zero/1.0/) applies to the data made available in this article, unless otherwise stated in a credit line to the data. 


\section{Background}

The side effects of cancer therapy have recently attracted significant attention [1]. Earlier diagnosis and improved systemic therapies, including targeted therapies, lead to longer life expectancy after cancer treatment [2-4]. Treatment-related comorbidities have thus become an important issue for long-term cancer survivors [5].

Cancer therapy-related cardiac dysfunction (CTRCD) is the second leading cause of death in cancer survivors after cancer-related mortality $[1,5,6]$. In particular, CTRCD is an important problem in the context of breast cancer because the incidence in young women is increasing while the prognosis is improving due to the development of various new treatments. Moreover, various breast cancer treatments specifically lead to cardiac dysfunction.

Current guidelines consider left ventricular ejection fraction (LVEF) assessment using echocardiography as the standard diagnostic technique for detecting chemotherapy-induced cardiotoxicity [7, 8]. However, magnetic resonance imaging (MRI) may play an important role in cardiac evaluation among cancer patients. MRI is the gold standard for the evaluation of ventricular volumes and function, with greater intra- and interobserver reproducibility than other modalities, and MRI may achieve higher sensitivity in identifying cardiomyopathy than other diagnostic techniques $[8,9]$.

The increasingly used T1 mapping sequences improved the usefulness of cardiac MRI, which enables tissue characterization through quantitative analysis of phenomena such as myocardial edema, inflammation, and fibrosis, thus playing an important role in the diagnosis of early and late cardiotoxicity in cancer patients $[10,11]$. However, the relationship between contractile dysfunction and microstructural injury is poorly understood. A previous study based on experiments on animals reported that T1-mapping MRI is useful for the early diagnosis of chemotherapyinduced cardiotoxicity [12].

The objective of our proposed study is to investigate the use of cardiac MRI to evaluate early tissue changes and perform functional assessment in chemo- and radiation-induced cardiotoxicity and to identify cardiotoxicity prognostic factors in patients with breast cancer.

\section{Primary hypothesis}

The Cardiac Magnetic Resonance for Early Detection of Cardiotoxicity in Breast Cancer (CareBest) study hypothesizes that the evaluation of early tissue changes and functional assessment using cardiac MRI would be useful in the early detection and risk stratification of chemotherapy- and radiation therapy-induced cardiotoxicity in patients with breast cancer.

\section{This hypothesis is supported by the following considerations}

1) In the early phase of exposure to an anthracycline agent, tissue injury and concurrent elevation of native $\mathrm{T} 1$ and extracellular volume fraction (ECV) values were noted in the preclinical study, without significant left ventricular (LV) reduction. Therefore, T1 mapping MRI allows for earlier detection of chemotherapy-induced cardiotoxicity [12]. T1 mapping parameters represent good surveillance markers of tissue injury during the early phase of cardiotoxicity.

2) Many studies suggest that CTRCD is not a functional but a structural disorder. The relationship between contractile dysfunction and microstructural injury, such as myocyte damage, fibrosis, and inflammation, remains poorly understood [11]. It was thus hypothesized that the evaluation of tissue changes using cardiac MRI would be more effective for cardiotoxicity risk stratification than the evaluation of functional changes.

\section{Methods and design \\ Overall study design}

CareBest is a single-center, large-scale prospective study. To achieve the goal of the study, cardiac MRI protocols for cardiotoxicity diagnosis will be applied to breast cancer patients who have received or are planning to receive chemotherapy. A 3-min cardiac imaging protocol was added to the breast MRI scanning protocol as a screening tool. The protocol includes T1 and T2 mapping and cine imaging of the LV myocardium. MRI is performed at 3 months, 6 months, and 2 years after treatment. Treatment protocols and MRI timing are shown in Fig. 1.

\section{Primary and secondary objectives}

The primary objective of this study is to investigate and compare the prognostic value of cardiac MRI parameters (native and postcontrast T1 mapping, ECV, right ventricular (RV), LV systolic function, and global longitudinal strain of LV) for cardiotoxicity. The endpoint is MACEs.

The secondary objectives are to determine the temporal relationships between contractile dysfunction and microstructural injury over a four-year period using cardiac MRI across all patients and by subgroup analysis according to treatment (e.g., chemotherapy regimen, targeted therapy agents, and radiation dose) and to compare the diagnostic accuracy of MRI, echocardiography or multigated acquisition (MUGA) by subgroup analysis. 


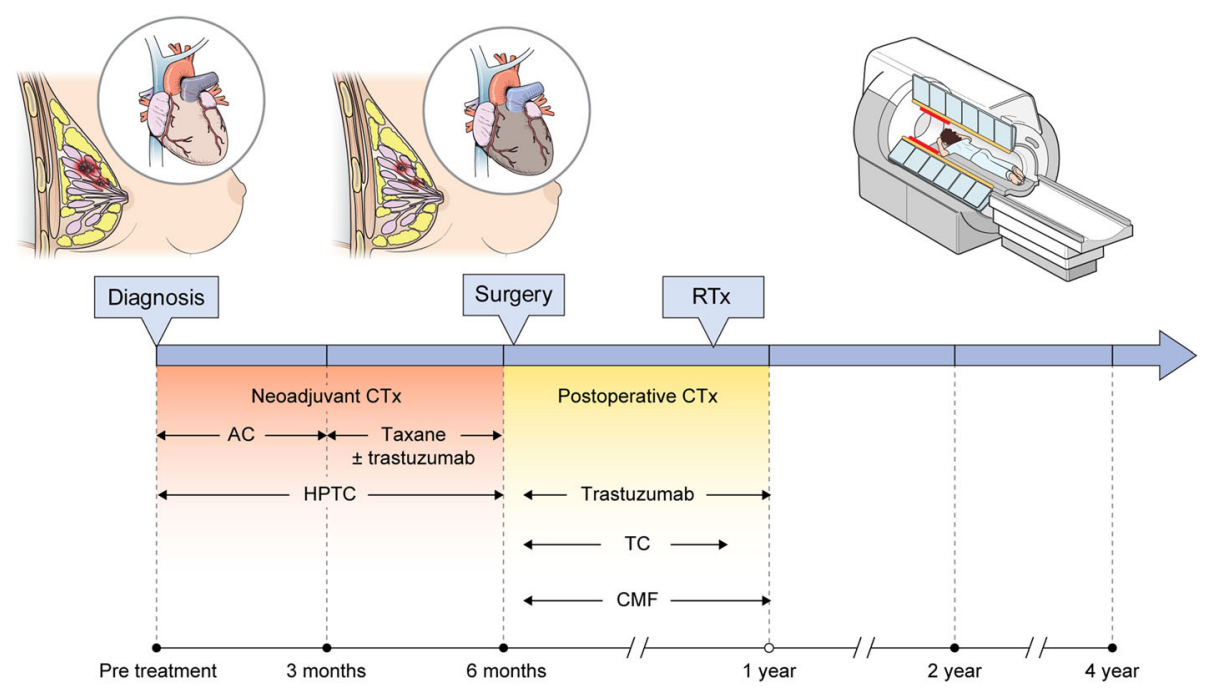

Fig. 1 Study design Black circles indicate MRI scanning

\section{Study population}

This prospective study will enroll over 2000 participants referred for breast cancer evaluation using MRI in a single center. All enrolled patients will undergo standardized breast MRI including a cardiac imaging protocol at the time points specified above and blood analysis to assess hematocrit (Hct) levels immediately before each MRI examination.

\section{Inclusion criteria}

Patients $\geq 18$ years of age are considered eligible if, following the initial oncology consultation, breast cancer is diagnosed and related therapeutic plans are formulated. All subjects will agree to participate by giving both verbal and written informed consent. This prospective study was approved by the institutional review board of Yonsei University College of Medicine.

Exclusion criteria include prior mammoplasty, history of other cancers, confirmed benign breast disease, poor image quality due to uncontrolled breast holding, arrhythmia, or patients with contraindications to MRI (i.e., claustrophobia, estimated glomerular filtration rate $($ eGFR $)<30)$.

\section{Cardiac MRI protocol}

A standardized breast and cardiac MR examination will be performed with $3 \mathrm{~T}$ MR scanners with an 8-channel breast coil (GE MRI 750w, GE Healthcare, Milwaukee, WI, USA). Because breast MRI is performed with the patient in a prone position, peripheral pulse gating will be applied for cardiac imaging.

The cardiac protocol includes pre- and postcontrast T1 mapping, T2 mapping, and cine imaging of both ventricular myocardia. Motion-corrected native $\mathrm{T} 1$ and postcontrast T1 and T2 mapping images will be acquired at the end of diastole in the short axis orientation of mid LV using a FIESTA-based 3(3)5 MOLLI sequence. Post-contrast T1 images will be acquired $15 \mathrm{~min}$ after the bolus injection of the contrast agent $(0.1 \mathrm{mmol} /$ $\mathrm{kg}$ body weight of gadolinium-based contrast agent, Gadobutrol $604.76 \mathrm{mg} / \mathrm{ml})$.

The imaging parameters for the 2D FIESTA cardiac cine MRI will be set as follows: flip angle $=45^{\circ}$, repetition time $(\mathrm{TR})=3.0 \mathrm{~ms}$, echo time $(\mathrm{TE})=1.1 \mathrm{~ms}$, field of view $($ FOV $)=390 \times 390 \mathrm{~mm}$, matrix $=160 \times 192$, measured pixel size $=2.4 \times 2.0 \mathrm{~mm}$, slice thickness $=8 \mathrm{~mm}(2$ $\mathrm{mm}$ gap between adjacent slices), number of slices $=9$, temporal resolution $=60 \mathrm{~ms}$, and ASSET factor $=2$.

The specific scan parameters for $\mathrm{T} 1$ mapping will be set as follows: flip angle $=35^{\circ}, \mathrm{TR}=2.8 \mathrm{~ms}, \mathrm{TE}=0.9 \mathrm{~ms}$, FOV $=300 \times 300 \mathrm{~mm}$, matrix $=160 \times 128$, delay time $(\mathrm{TD})=220 \mathrm{~ms}$, measured pixel size $=1.9 \times 2.3 \mathrm{~mm}$, slice thickness $=8 \mathrm{~mm}$, and acquisition window $=361 \mathrm{~ms}$.

The specific scan parameters for FSE-based black blood T2 mapping will be set as follows: $T R=1 \mathrm{RR}$; total echo train length $(\mathrm{ETL})=16 ; \mathrm{FOV}=300 \times 300 \mathrm{~mm}$; matrix $=$ $160 \times 128$; measured pixel size $=1.9 \times 2.3 \mathrm{~mm}$; slice thickness $=8 \mathrm{~mm}$; 4 echoes with effect TEs $=11.3,33.9,56.5$, and $79.1 \mathrm{~ms}$; acquisition window $=91 \mathrm{~ms}$; and ASSET factor $=2$.

Sequential short axis cine imaging will be performed including the entire ventricular myocardium in addition to standard long-axis views using the 2D FIESTA sequence. The specific scan parameters will be set as follows: flip angle $=45^{\circ}, \mathrm{TR}=3.0 \mathrm{~ms}, \mathrm{TE}=1.1 \mathrm{~ms}$, FOV $=$ $390 \times 390 \mathrm{~mm}$, matrix $=160 \times 192$, measured pixel size $=$ $2.4 \times 2.0 \mathrm{~mm}$, slice thickness $=8 \mathrm{~mm}(2 \mathrm{~mm}$ gap between adjacent slices), number of slices $=9$, temporal resolution $=60 \mathrm{~ms}$, and ASSET factor $=2$. 
The sequences for breast MR examination include a three-plane localizing sequence, axial T2-weighted fastspin- echo and T2-stimulated inversion recovery (STIR) sequence, and diffusion-weighted imaging before contrast administration. 3D dynamic postcontrast-enhanced (DCE) images and a T1-weighted 3D delayed postcontrast sequence will be acquired in the sagittal plane after contrast injection.

Native T1 and T2 images will be acquired between T2-STIR and diffusion images, while postcontrast T1 images will be acquired after delayed postcontrast images. Cine images will be acquired before dynamic images. Precontrast T1 and T2 mapping images will be acquired before DWI imaging, and postcontrast T1 mapping imaging will be performed after dynamic contrast imaging. The hematocrit (Hct) levels of the patients will be acquired on the day of cardiac MRI.

\section{Cardiac MRI analysis}

\section{Both ventricular volumes and systolic function}

All MR images will be analyzed using $\mathrm{cvi}^{42}$ image analysis software (Circle Cardiovascular Imaging Inc., Calgary, $A B$, Canada). Short-axis cine images will be analyzed using semi-automated contouring of the endocardial and epicardial borders of both ventricles at enddiastole and end-systole to calculate both ventricular function (i.e., RV, LV end-diastolic volume (RVEDV, LVEDV), end-systolic volume (RVESV, LVESV), cardiac output, stroke volume, RVEF, and LVEF).

\section{$T 1$ and $T 2$ mapping analysis}

One native T1, one postcontrast T1, and one T2 mapping image acquired on the mid LV will be transferred to the software. A region of interest (ROI) will be freely drawn on the septum. Images with severe artifacts will be coded and removed from the analysis.

Mean segmental native T1, T2, and postcontrast T1 values are calculated for each patient. ECV values are calculated using the mean values of native and postcontrast $\mathrm{T} 1$ and hematocrit, as previously established [13].

\section{Myocardial strain analysis}

Feature tracking analysis will also be performed using cvi42 software. The long axis and short-axis cine images will be loaded onto the software. Endocardial and epicardial borders of the LV in both the long- and short-axis views were semi-automatically delineated in the enddiastolic phase. The feature software automatically measured the global longitudinal strain in the twodimensional (2D) longitudinal directions. Analysis of interobserver agreement in regard to MRI measurements will be performed.

\section{Chemotherapy regimen \\ Cytotoxic chemotherapy}

- AC regimen: doxorubicin $60 \mathrm{mg} / \mathrm{m}^{2}$ with cyclophosphamide $\left(600 \mathrm{mg} / \mathrm{m}^{2}\right)$ IV every 3 weeks for a total of 4 cycles (cumulative dose of doxorubicin: $240 \mathrm{mg} / \mathrm{m}^{2}$ )

- AC followed by taxane: 4 cycles of AC followed by taxane (paclitaxel $80 \mathrm{mg} / \mathrm{m}^{2}$ IV once a week for 12 weeks, or docetaxel $75 \mathrm{mg} / \mathrm{m}^{2}$ IV every 3 weeks, for a total of 4 cycles)

- CMF regimen: cyclophosphamide $\left(600 \mathrm{mg} / \mathrm{m}^{2}\right)$ with methotrexate $\left(60 \mathrm{mg} / \mathrm{m}^{2}\right)$ and fluorouracil $(600 \mathrm{mg} /$ $\mathrm{m}^{2}$ ) at days 1 and 8 every 4 weeks, for a total of 6 cycles

- TC regimen: docetaxel $75 \mathrm{mg} / \mathrm{m}^{2}$ and cyclophosphamide $600 \mathrm{mg} / \mathrm{m}^{2}$ IV every 3 weeks for a total of 4 cycles

\section{HER2-targeted therapy}

-HPTC regimen: Trastuzumab $8 \mathrm{mg} / \mathrm{kg}$ loading dose and $6 \mathrm{mg} / \mathrm{kg}$ maintenance dose, pertuzumab $840 \mathrm{mg}$ loading dose and $420 \mathrm{mg}$ maintenance dose, docetaxel $75 \mathrm{mg} /$ $\mathrm{m}^{2}$, carboplatin every 3 weeks for a total of 6 cycles.

-Trastuzumab: trastuzumab $8 \mathrm{mg} / \mathrm{kg}$ IV (loading dose) followed by $6 \mathrm{mg} / \mathrm{kg}$ IV (maintenance dose) every 3 weeks for a total of 18 cycles or trastuzumab $600 \mathrm{mg}$ SC every 3 weeks for a total of 18 cycles for HER2-positive breast cancer: Trastuzumab will be given after the completion of the doxorubicin regimen regardless of the taxane regimen.

\section{Clinical outcomes}

The primary outcome is MACEs defined as CV death, hospitalization due to heart failure, and/or heart transplantation. The secondary outcome is left ventricular dysfunction, defined as a decrease in the LVEF of $>10$ percentage points to a value $<53 \%$ [9]. The follow-up duration will be up to 4 years after treatment.

\section{Sample size considerations}

There is no generally accepted approach to estimate the sample size for studies of risk prediction models [14]. We will enroll the largest possible number of patients to ensure the stability of the prediction model based on MRI parameters.

\section{Preliminary study}

Reliability of cardiac imaging during breast MRI

We will perform a preliminary study to evaluate the feasibility of cardiac imaging during breast MRI in control subjects $(n=15)$. All patients will undergo planned cardiac imaging with a cardiac coil in a supine position, without contrast agent. A native T1 cardiac image in a 
short axis plane will be acquired. After cardiac imaging, the planned breast+cardiac imaging protocol will be performed in a prone position with contrast agent.

\section{Statistical analysis}

Categorical baseline characteristics will be expressed as numbers and percentages, and continuous variables will be expressed as the means and standard deviations. To evaluate the prognostic value of T1 mapping parameters for cardiotoxicity, Cox proportional hazard regression or other transformations will be used after checking the proportional hazard assumption. To determine and compare the prognostic value of native $\mathrm{T} 1$ and $\mathrm{ECV}$ and that of LVEF or global longitudinal strain of LV, timedependent ROC curves, Harrell's $\mathrm{C}$ statistic and bootstrapping with 1000 repetitions will be used. A linear mixed model will be used to evaluate the temporal relationship between LVEF, native T1, ECV, and T2 values. The diagnostic accuracies of MRI, MUGA, and echocardiography for cardiotoxicity will be evaluated by ROC curves, and the significance of the differences among the related areas under the curves (AUC) will be evaluated using Delong's method [15]. P-values less than 0.05 will be considered statistically significant. All statistical analyses will be performed using SAS (version 9.4, SAS Institute Inc., Cary, NC, USA).

\section{Study approval and progression}

The study protocol was approved by the regional institutional review board in October 2016 (IRB No. 4-20160730), and the protocol is registered at clinicaltrials.gov (Clinical trial no. NCT03301389). A data and safety monitoring board was constituted to ensure the safe continuation of the study.

\section{Discussion}

This prospective study will be the largest study investigating serial MRI-based evaluation of CTRCD. We will investigate the effects of chemotherapy, radiation therapy, and other therapies on myocardial function and structure, thus providing additional evidence on whether cardiac MRI is the optimal screening tool for the diagnosis of chemo or radiation therapy-induced cardiotoxicity, given its advantages in terms of quantitative assessment.

Recently, significant attention has been focused on the field of cardio-oncology. Many influential clinical guidelines for cardiovascular toxicity have been developed [8, 10, 16-19]. The current diagnostic criteria are based on left ventricular functional changes [17]. However, chemotherapy-induced cardiotoxicity is considered a continuum that begins with subclinical myocardial cell injury, leading to an early, asymptomatic decline in LVEF, which can eventually develop into symptomatic heart failure [12, 20]. LVEF can be used to detect myocardial damage only after functional impairment has already occurred.

Cardiac MRI is the gold standard for the evaluation of ventricular volumes and function, with greater intraand inter-observer reproducibility than other methods, and quantitative cardiac MRI may play an important role in the cardiac evaluation and risk stratifications of cancer patients [11, 21]. Recently, many papers have been published that show the usefulness of MRI-obtained parameters such as ventricular volume, function, mass, and strain in diagnosing CTRCD [11, 22-26]. The most important capability of MRI is tissue characterization with quantitative MR parameters. Tissue characterization by cardiac MRI can help identify early myocardial injuries that are not included in the current diagnostic criteria $[11,21,27]$.

Breast cancer therapy involves many adjuvant therapies in addition to surgery, most of which cause heartrelated problems. Anthracycline, used as a primary chemotherapy agent in breast cancer, is a well-known cardiotoxic agent $[8,28]$, with the incidence of cardiotoxicity ranging from 4 to $>36 \%$ in patients receiving $500-550 \mathrm{mg} / \mathrm{m}^{2}$.

Other conventional chemotherapy agents used in breast cancer treatment, including cyclophosphamide and taxanes, also induce myocardial dysfunction [8]. The recently adopted targeted therapy agent trastuzumab, which improves outcomes of patients with HER2positive breast cancer, is also notoriously associated with treatment-related cardiotoxicity [8].

Radiation therapy, another major treatment for breast cancer, induces myocardial fibrosis, microcirculatory injury, and myocardial infarction. High-dose radiation exposure is used on the thorax in the adjuvant setting after breast surgery [29].

Therefore, cardiac evaluation during breast cancer treatment is clinically very important. This study is based on the observation that it is very efficient to perform an additional myocardium examination during the routine surveillance of breast cancer. Indeed, the separate assessment of cardiac function using echocardiography or MUGA on a regular basis during surveillance of breast cancer treatment is somewhat cumbersome. Moreover, serial MUGA evaluations may cause significant radiation exposure. For these reasons, we set up a short-term imaging protocol for the evaluation of myocardial tissue and myocardial dysfunction, and we tested the feasibility of cardiac imaging using a breast coil in the preliminary study.

The limitation of this study is that it is a single-center prospective registry study. Although blood biomarkers such as NT proBNP or troponins have been demonstrated to be good early markers of cardiotoxicity, they are not included in the study protocol. 
In conclusion, the simultaneous evaluation of breast cancer and cardiac function during treatment can potentially be very effective in terms of cost and time. Moreover, it may help the early detection of cardiotoxicity during cancer therapy, while quantitative imaging methods may provide additional value for the surveillance of patients at risk of CTRCD.

\section{Abbreviations}

MRI: Magnetic resonance imaging; LV: Left ventricular; RV: Right ventricle; EF: Ejection fraction; LVEF: Left ventricular ejection fraction; ECV: Extracellular volume fraction; MACE: Major adverse cardiac event; MUGA: Multigated acquisition; CTRCD: Cancer therapy-related cardiac dysfunction; eGFR: Estimated glomerular filtration rate; STIR: Stimulated inversion recovery; DCE: Dynamic postcontrast enhanced; Hct: Hematocrit; LVEDV, RVEDV: LV or RV end-diastolic volume; LVESV, RVESV: LV or RV end-systolic volume; ROI: Region of interest; AUC: Area under the curve; ICC: Intraclass correlation coefficient

\section{Acknowledgements}

The authors would like to thank MID (Medical Illustration \& Design), a part of the Medical Research Support Services of Yonsei University College of Medicine, for all artistic support related to this work and the research team at GE Healthcare Korea for the technical support for T1 mapping imaging.

\section{Consent for publications}

Not applicable.

\section{Authors' contributions}

YJH, GMK, SAL, MJK, and BWC are participating in the patient enrollment. YJH, GMK, MJK, and BWC are participating in the study design. PKK set up the MRI protocol. YJH, GMK, JH, YJK, HJL, and BWC are editing the manuscript. EA and JYL are managing the data. $\mathrm{KH}$ is participating in sample size calculation and statistical analysis. All authors have read and approved the manuscript.

\section{Funding}

This work was supported by the National Research Foundation of Korea (grant NRF-2017R1A2B4009661) and by the Basic Science Research Program through the National Research Foundation of Korea, funded by the Ministry of Science, Information and Communication Technology, and Future Planning (grant NRF-2014R1A1A3050905) and faculty research grant of Yonsei University College of Medicine (6-2016-0077).

These funding bodies only provide funding and played no role in the research design; data collection, analysis and interpretation; or the writing of the manuscript.

\section{Availability of data and materials}

Not applicable.

\section{Ethics approval and consent to participate}

The study protocol was approved by the regional institutional review board (IRB No. 4-2016-0730).

\section{Competing interests}

The authors declare that they have no competing interests.

\section{Author details}

1Department of Radiology and Research Institute of Radiological Science, Severance Hospital, Yonsei University College of Medicine, 50 Yonsei-ro, Seodaemun-gu, Seoul 120-752, South Korea. ${ }^{2}$ Department of Internal Medicine, Yonsei University College of Medicine, Seoul, South Korea.

Received: 28 January 2020 Accepted: 27 April 2020

Published online: 03 June 2020

\section{References}

1. Lenneman CG, Sawyer DB. Cardio-oncology: an update on Cardiotoxicity of Cancer-related treatment. Circ Res. 2016;118(6):1008-20.
2. Bovelli D, Plataniotis G, Roila F, Group EGW. Cardiotoxicity of chemotherapeutic agents and radiotherapy-related heart disease: ESMO Clinical Practice Guidelines. Ann Oncology. 2010;21(suppl_5):v277-v82.

3. Siegel R, DeSantis C, Virgo K, Stein K, Mariotto A, Smith T, et al. Cancer treatment and survivorship statistics, 2012. CA Cancer J Clin. 2012;62(4): 220-41.

4. Trama A, Botta L, Foschi R, Ferrari A, Stiller C, Desandes E, et al. Survival of European adolescents and young adults diagnosed with cancer in 2000-07: population-based data from EUROCARE-5. Lancet Oncol. 2016;17(7):896-906.

5. Felker GM, Thompson RE, Hare JM, Hruban RH, Clemetson DE, Howard DL, et al. Underlying causes and long-term survival in patients with initially unexplained cardiomyopathy. N Engl J Med. 2000;342(15):1077-84.

6. Abdel-Qadir H, Austin PC, Lee DS, Amir E, Tu JV, Thavendiranathan P, et al. A population-based study of cardiovascular mortality following early-stage breast Cancer. JAMA Cardiol. 2017;2(1):88-93.

7. Colombo A, Sandri MT, Salvatici M, Cipolla CM, Cardinale D. Cardiac complications of chemotherapy: role of biomarkers. Curr Treat Options Cardiovasc Med. 2014;16(6):313.

8. Zamorano JL, Lancellotti P, Rodriguez Munoz D, Aboyans V, Asteggiano R, Galderisi M, et al. 2016 ESC position paper on cancer treatments and cardiovascular toxicity developed under the auspices of the ESC Committee for practice guidelines: the task force for cancer treatments and cardiovascular toxicity of the European Society of Cardiology (ESC). Eur Heart J. 2016;37(36):2768-801.

9. Plana JC, Galderisi M, Barac A, Ewer MS, Ky B, Scherrer-Crosbie M, et al. Expert consensus for multimodality imaging evaluation of adult patients during and after cancer therapy: a report from the American Society of Echocardiography and the European Association of Cardiovascular Imaging. Eur Heart J Cardiovasc Imaging. 2014;15(10):1063-93.

10. Bloom MW, Hamo CE, Cardinale D, Ky B, Nohria A, Baer L, et al. Cancer therapy-related cardiac dysfunction and heart failure: part 1: definitions, pathophysiology, risk factors, and imaging. Circ Heart Fail. 2016;9(1): e002661.

11. Thavendiranathan P, Wintersperger BJ, Flamm SD, Marwick TH. Cardiac MRI in the assessment of cardiac injury and toxicity from cancer chemotherapy: a systematic review. Circ Cardiovasc Imag. 2013;6(6):1080-91.

12. Hong YJ, Park HS, Park JK, Han K, Park CH, Kim TK, et al. Early detection and serial monitoring of Anthracycline-induced Cardiotoxicity using T1mapping cardiac magnetic resonance imaging: an animal study. Sci Rep. 2017;7(1):2663.

13. Messroghli DR, Moon JC, Ferreira VM, Grosse-Wortmann L, He T, Kellman P, et al. Clinical recommendations for cardiovascular magnetic resonance mapping of T1, T2, T2* and extracellular volume: a consensus statement by the Society for Cardiovascular Magnetic Resonance (SCMR) endorsed by the European Association for Cardiovascular Imaging (EACVI). J CardiovasC Magn Reson. 2017;19(1):75

14. Moons KG, Altman DG, Reitsma JB, loannidis JP, Macaskill P, Steyerberg EW, et al. Transparent reporting of a multivariable prediction model for individual prognosis or diagnosis (TRIPOD): explanation and elaboration. Ann Intern Med. 2015;162(1):W1-73.

15. DeLong ER, DeLong DM, Clarke-Pearson DL. Comparing the areas under two or more correlated receiver operating characteristic curves: a nonparametric approach. Biometrics. 1988;44(3):837-45.

16. Zamorano JL, Lancellotti $P$, Rodriguez Munoz D, Aboyans V, Asteggiano R, Galderisi M, et al. 2016 ESC position paper on cancer treatments and cardiovascular toxicity developed under the auspices of the ESC Committee for practice guidelines: the task force for cancer treatments and cardiovascular toxicity of the European Society of Cardiology (ESC). Eur Heart Fail. 2017;19(1):9-42.

17. Hamo CE, Bloom MW, Cardinale D, Ky B, Nohria A, Baer L, et al. Cancer therapy-related cardiac dysfunction and heart failure: part 2: prevention, treatment, guidelines, and future directions. Circ Heart Fail. 2016;9(2); e002843.

18. Armenian SH, Hudson MM, Mulder RL, Chen MH, Constine LS, Dwyer M, et al. Recommendations for cardiomyopathy surveillance for survivors of childhood cancer: a report from the international late effects of childhood Cancer guideline harmonization group. Lancet Oncol. 2015;16(3):e123-36.

19. Curigliano G, Cardinale D, Suter T, Plataniotis G, de Azambuja E, Sandri MT, et al. Cardiovascular toxicity induced by chemotherapy, targeted agents and radiotherapy: ESMO Clinical Practice Guidelines. Ann Oncol. 2012;23(Suppl 7):vii155-66. 
20. Cardinale D, Colombo A, Bacchiani G, Tedeschi I, Meroni CA, Veglia F, et al. Early detection of anthracycline cardiotoxicity and improvement with heart failure therapy. Circulation. 2015;131(22):1981-8.

21. Jordan JH, Todd RM, Vasu S, Hundley WG. Cardiovascular magnetic resonance in the oncology patient. JACC Cardiovasc Imaging. 2018;11(8): 1150-72.

22. Galan-Arriola C, Lobo M, Vilchez-Tschischke JP, Lopez GJ, de Molina-Iracheta $A$, Perez-Martinez $C$, et al. Serial magnetic resonance imaging to identify early stages of Anthracycline-induced Cardiotoxicity. J Am Coll Cardiol. 2019;73(7):779-91.

23. Ferreira de Souza T, Quinaglia ACST, Osorio Costa F, Shah R, Neilan TG, Velloso L, et al. Anthracycline therapy is associated with Cardiomyocyte atrophy and preclinical manifestations of heart disease. JACC CardiovasC Imaging. 2018;1 1(8):1045-55.

24. Gong IY, Ong G, Brezden-Masley C, Dhir V, Deva DP, Chan KKW, et al. Early diastolic strain rate measurements by cardiac MRI in breast cancer patients treated with trastuzumab: a longitudinal study. Int J Cardiovasc Imag. 2019; 35(4):653-62.

25. Barthur A, Brezden-Masley C, Connelly KA, Dhir V, Chan KK, Haq R, et al. Longitudinal assessment of right ventricular structure and function by cardiovascular magnetic resonance in breast cancer patients treated with trastuzumab: a prospective observational study. J Cardiovasc Magn Reson. 2017;19(1):44.

26. Ong G, Brezden-Masley C, Dhir V, Deva DP, Chan KKW, Chow CM, et al. Myocardial strain imaging by cardiac magnetic resonance for detection of subclinical myocardial dysfunction in breast cancer patients receiving trastuzumab and chemotherapy. Int J Cardiol. 2018;261:228-33.

27. Plana JC, Thavendiranathan P, Bucciarelli-Ducci C, Lancellotti P. Multimodality imaging in the assessment of cardiovascular toxicity in the Cancer patient. JACC Cardiovasc Imaging. 2018;11(8):1173-86.

28. Henry ML, Niu J, Zhang N, Giordano SH, Chavez-MacGregor M. Cardiotoxicity and cardiac monitoring among chemotherapy-treated breast Cancer patients. JACC Cardiovasc Imaging. 2018;11(8):1084-93.

29. Key TJ, Verkasalo PK, Banks E. Epidemiology of breast cancer. Lancet Oncol. 2001;2(3):133-40.

\section{Publisher's Note}

Springer Nature remains neutral with regard to jurisdictional claims in published maps and institutional affiliations.

Ready to submit your research? Choose BMC and benefit from:

- fast, convenient online submission

- thorough peer review by experienced researchers in your field

- rapid publication on acceptance

- support for research data, including large and complex data types

- gold Open Access which fosters wider collaboration and increased citations

- maximum visibility for your research: over $100 \mathrm{M}$ website views per year

At $\mathrm{BMC}$, research is always in progress.

Learn more biomedcentral.com/submissions 